\title{
Determinantes socioeconómicos de la posesión de automóviles en los municipios de México ${ }^{\mathrm{T}}$
}

\author{
Socioeconomic determinants \\ of car ownership in Mexican Municipalities \\ Ignacio Javier Cruz Rodríguez \\ Cátedras Consejo Nacional de Ciencia y Tecnología \\ Centro de Investigaciones Socioeconómicas de la \\ Universidad Autónoma de Coahuila, Saltillo, México \\ ORCID: http://orcid.org/oooo-0003-4889-5838 \\ DoI: http://dx.doi.org/I0.28928/revistaiztapalapa/842018/aot2/cruzrodriguezij
}

\section{Resumen}

El principal objetivo de este artículo es cuantificar los impactos de variables socioeconómicas en la posesión de vehículos en los municipios de México para el año 20Io. Se separaron los municipios es urbanos y rurales y se tomó la información del censo económico para estimar los impactos en cada uno. Los resultados indican que el ingreso es la variable de mayor impacto en la posesión, de hecho, es de mayor magnitud que las estimaciones encontradas en la literatura alrededor del mundo. Otras variables de importancia son la densidad de población y la provisión de vialidades que ofrece evidencia de tráfico inducido. Los resultados son comentados en términos de su utilidad para la formulación de políticas.

Palabras clave: Posesión de automóvil, Municipios, México, movilidad, motorización.

\begin{abstract}
The main objective of this paper is to quantify de impacts of socioeconomics variables in car ownership in Mexican municipalities in 20I0. I separated urban and rural municipalities in order to appreciate different impacts. The results show that income per capita has the highest impact, in fact, is the grater than the others founded in literature around the world. Other important variables are population density and roadway provision. The roadway provision offers evidence of induced traffic. The results are commented in terms of public policy uses.
\end{abstract}

Key words: Car Ownership, Mexico, Municipalities, mobility, motorization.

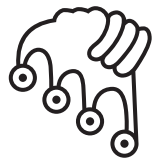

IZTAPALAPA

Agua sobre lajas

1 El autor agradece los comentarios de dictaminadores anónimos que contribuyeron a mejorar este documento. 


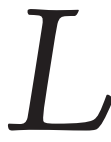

a propiedad o posesión de un vehículo automotor es un rasgo característico de las sociedades contemporáneas. Dicha posesión es una decisión que toman de los agentes económicos que tiene importantes consecuencias para los individuos pues incrementa dramáticamente su movilidad y le da acceso a oportunidades que no tendría de otro modo como el empleo, además de que puede ser símbolo de estatus social (Rooda 1998: I). De hecho, existe evidencia de González-Arellano (2013), que afirma que la posesión y el uso del auto han transformado el territorio que, a su vez, ha modificado tanto distancias como el acceso a oportunidades que no se tienen al interior de las ciudades si no se cuenta con automóvil. En este sentido la posesión del automóvil ha modificado fuertemente la movilidad.

Aunque la posesión de un automóvil no es lo mismo que el uso del mismo, es muy importante mencionar que ambas variables están estrechamente asociadas y es muy fácil tomarlas como sinónimos o como variables proxy una de la otra. De dicha posesión y/o uso se desprenden problemas que incluyen dependencia del consumo de energía, congestión vial, emisiones de gases contaminantes, contaminación auditiva, seguridad vial, entre otras. Tales consecuencias son normalmente abordadas como externalidades de cuyo estudio toma fuerte relevancia la posesión de automóviles.

En el artículo de Dargay et al., (2007:18), se estimó una proyección de posesión de vehículos con información agregada a nivel nacional para países de la OCDE para el periodo 1960-2030 en donde los autores pronosticaron que tanto en Turquía como en México la posesión de vehículos crecerá más rápido que el ingreso. Una referencia como la mencionada subraya la necesidad de conocer las variables que afectan la propiedad de vehículos. Para ello, este artículo se divide en seis secciones. En la segunda se revisa brevemente el aspecto teórico relacionado con la posesión de vehículos para dar paso a la tercera sección en la que se analiza la literatura encontrada respecto de la posesión de vehículos. La cuarta aborda el análisis estadístico de los datos mientras que la quinta sección se enfoca en el modelo econométrico y en la última se presentan las conclusiones. 


\section{Breve revisión teórica}

La posesión de un vehículo automotor o automóvil, al igual que otros bienes en una economía, está precedida de la decisión de compra por parte de los consumidores. La diferencia entre adquirir un automóvil y cualquier otro bien radica en varios hechos. En primer lugar, es un bien de consumo duradero en el sentido de que su uso puede continuar a lo largo de cierto periodo de tiempo ${ }^{2}$. La diferencia con otro tipo de bienes es que la decisión por adquirirlos está basada en necesidades diferentes a las que se suele argumentar para determinar la compra de bienes no durables como la comida, que se basa en la necesidad de alimentación. Por tanto, el ingreso es considerado como una variable de fundamental importancia para entender la posesión de automóviles. Profundizando, los aportes teóricos como el de Sun et al. (2015:3), indican que el efecto en la posesión será positivo, pero tenderá a disminuir a medida que las personas posean previamente uno o más automóviles como resultado de que han cubierto la necesidad de propiedad.

Desde un punto de vista microeconómico y asociado al tema del párrafo anterior, la posesión de automóviles está asociada a la existencia de un bien que pueda ser considerado sustituto. En este sentido toma relevancia la provisión de transporte público. Los atributos que tenga el bien sustituto como calidad, comodidad, accesibilidad y seguridad pueden determinar en buena medida la posesión de un vehículo. Un elemento extra que se considera importante es el costo de transporte, es decir, la erogación que realiza un usuario por utilizar el transporte que incluye combustible y lubricantes, compra de un seguro, costos de estacionamiento entre otros, para el caso de tener automóvil y su comparación con la erogación que realizaría por usar una modalidad pública. Según De Rus (2003: 432), el reparto entre transporte público y privado de la cantidad de pasajeros está determinado por diversos factores entre los que se encuentran el costo por kilómetro de utilizar el automóvil (gastos de combustible), las velocidades respectivas del transporte público y privado (en la que se puede resaltar la existencia de tráfico), el valor del tiempo de traslado para los individuos y una utilidad de reserva que se supone suficientemente alta para que siempre se lleve a cabo un desplazamiento. De acuerdo con los valores que tome una función de utilidad los individuos decidirán poseer y utilizar un automóvil o el transporte público. Cuando los costos de usar el automóvil junto con su tiempo

2 El Instituto Nacional de Estadística Geografía e Informática (INEGI) considera que al automóvil un bien de consumo duradero del nivel más alto. INEGI (20I5:46) 
de traslado son mayores al que reportaría el uso de transporte público existirá un incentivo para que los usuarios usen transporte público y viceversa.

Un argumento que resulta fundamental en la posesión de un vehículo es la distancia que se pretenda recorrer con el automóvil ya que ésta determinará costos de combustible y tiempo de estancia dentro del automóvil. Como lo mencionan Sun et al. (2005:2), la provisión de infraestructuras de tránsito y el nivel de urbanización forman parte de la estructura urbana.

Dentro de tal estructura urbana se encuentran concentrados tanto los orígenes como los destinos de los viajes que se realizan al interior de ella correspondiéndoles una distancia asociada a cada viaje y con ello un tiempo de traslado necesario para recorrerla. En este mismo sentido el modelo de Handy et al., (2005), predice que, si la extensión de las vialidades es larga y, además, el crecimiento de una ciudad es constante será más probable encontrar altas tasas de posesión de vehículos. Una relación que se desprende de la existencia de vialidades y posesión de automóviles es la conocida como tráfico inducido. De acuerdo con Galindo (2006:146) los cambios en el tiempo de traslado, generados por nuevas vialidades, se traducirán en modificaciones de las rutas, horarios, número de viajes y kilómetros recorridos pues la reducción en el tiempo del trasportarse o en los costos que implica un incrmento en el uso de vialidades.

Un último aspecto por considerar es la estructura social y familiar. Existen visiones teóricas que resaltan la importancia de la estructura familiar, la edad, el género, entre otras variables en la propiedad vehicular. La edad juega uno de los papeles más importantes ya que las personas adultas son las que forman, en su mayoría, la movilidad de una ciudad. Sus desplazamientos se encuentran influenciados por el empleo, diversión y esparcimiento, hogar y escuela principalmente. Es por ello que el número de adultos en una demarcación puede influenciar fuertemente a la posesión vehicular. Como lo demostró Matas y Raymond (2008) el aumento en las necesidades de viajes nombrado efecto generación tiene como resultado un incremento en la posesión durante los siguientes años.

\section{Revisión de literatura empírica}

En la literatura se encuentran dos tipos de modelo de determinantes de posesión o propiedad de vehículos: a nivel individual, cuya información proviene normalmente de una encuesta, y a nivel agregado, cuya información agregada está delimitada por una unidad político territorial. Esta investigación se alinea con la segunda opción 
mencionada pues la información utilizada se encuentra agregada para los municipios de México en el año 20ı. La heterogeneidad de la literatura revisada requiere de un criterio de clasificación por lo que se decidió agruparlas por variable explicativa y centrar los reportes de los estudios en el tamaño del impacto que tuvieron en la posesión. Se incluyó también una subclasificación por tipo de técnica econométrica utilizada con el fin de tener otra agrupación que permita una mejor identificación al momento de hacer los comparativos.

Antes de iniciar con la revisión conviene detenerse a reflexionar sobre las diferencias de contexto en la escala de análisis. Existen estudios de posesión de vehículos para diversas regiones del mundo cuyos resultados varían. El contexto en el que se dan dichos resultados resulta muy importante para entender las variaciones en los resultados. La idiosincrasia de los habitantes de cada país, los hábitos, entre otras razones afectan la posesión de automóviles. Por ejemplo, es bien sabido que la movilidad en ciudades europeas como Ámsterdam reportan un fuerte porcentaje de viajes en bicicleta que implica un menor uso del automóvil desincentivando la posesión. Por su parte, los estudios a nivel ciudad o grupo de ciudades agrupadas aterrizan un poco más el nivel de análisis que los respectivos a nivel nacional, pero incluso en esos niveles de análisis se corre el riesgo de no diferenciar la posesión en grandes y pequeñas urbes. Por tanto, resulta conveniente no perder de vista estas diferencias a la hora de comparar resultados.

Una primera variable que resulta fundamental para explicar la propiedad de vehículos es el ingreso. La principal característica de los estudios que versan sobre la relación entre ingreso y propiedad de autos es que todos coinciden en delimitar una zona político administrativa para llevar a cabo sus cálculos que van desde municipios y ciudades hasta países. La literatura es vasta y los casos aplicados existen alrededor del mundo. Es ampliamente aceptado que el ingreso es una razón primaria para la posesión de un vehículo. En modelos de demanda tanto agregada como individual se incluye como variable debido a que los individuos necesitan suficiente ingreso para comparar, mantener y conducir un carro como puede verse en los estudios de Nobile et al., (1995), para Holanda; Ryan y Han (1999), para Honolulu, Hawaii; Chingcuanco y Miller (2014), para el caso de la ciudad de Ontario; Xu et al., (2015), para la ciudad de Beijing; Cao et al., (2006), para el estado de Carolina del Norte; Hsu et al., (2007), para el caso de ciudades taiwanesas y Clark (2009), para Gran Bretaña. Para el caso específico de la Ciudad de México, el estudio de Guerra (2015), indica que la posesión de automóviles ha crecido al mismo tiempo que los suburbios. Este autor afirma que la flota de vehículos creció más rápidamente que la población entre los años 1980-2010. 
Macfarland y Garroe (2015), desagregan el impacto del ingreso en la posesión de dos y tres vehículos para trece condados en Atlanta, Georgia. Los hogares con mayor número de vehículos presentan una asociación positiva de la posesión de vehículos al ingreso pues su coeficiente asociado es de 0.452 mientras que el correspondiente a la posesión de dos vehículos es de 0.37I. Un impacto muy similar lo estimó Sun et al., (2015), aunque éste autor no utiliza información a nivel país, sino que agrega la información de I6I ciudades en China. Su estudio revela que el impacto del ingreso en la propiedad de vehículos es positivo (0.374) y estadísticamente significativo, aunque esta variable no resultó ser la de mayor impacto. Otro estudio para países de la OCDE, pero con diferente periodo de tiempo abarcado es el de Johnstone et al., (2009), que estiman que la relación tiene un impacto positivo y significativo cuyo valor del coeficiente estimado es de 0.02. Cabe señalar que dicho valor es el más pequeño de los que reportó la búsqueda en la literatura.

Dargay y Giuliano (2006), estiman el impacto del ingreso en la propiedad de vehículos para diversos rangos de ingreso en los Estados Unidos. Sus resultados indican que por debajo de cierto nivel de ingreso el coeficiente resulta negativo $y$ significativo (0.5I) pero para ingresos medianos cambia a positivo $(0.44)$ y para ingresos superiores se mantiene positivo y significativo (0.76).

No obstante que la evidencia apunta mayoritariamente a que el ingreso impacta positivamente a la posesión de autos, existen estudios como el de Huang (2OII), en el que la estimación reportada no presentó significancia estadística indicando que el ingreso no resultó importante para explicar la posesión.

Los documentos que se mencionan en adelante utilizaron una técnica econométrica alternativa. En primer lugar, Dargay y Gately (1999), reportan la estrecha relación entre estas variables para países de la OCDE. Los autores calculan las elasticidades de corto y largo plazo e indican que la propiedad crece al doble de lo que lo hace el ingreso. Sus conclusiones son soportadas con una estimación de curva de Gompertz. Dargay (2002), ofrece evidencia, mediante un panel de datos, del impacto que tiene la variable ingreso en el Reino Unido entre los años 1970 y 1995 cuyo cálculo de la elasticidad resulta positiva y estadísticamente significativa (0.4I8). Este autor es importante porque separa los datos en ambientes rurales y urbanos reportando como una de sus principales conclusiones que en ambientes rurales los costos asociados a la propiedad son mucho menos sensibles a su contraparte urbana.

Woldeamanuel et al., (2009), usando un panel de datos, realizaron un análisis para determinar los cambios en el comportamiento de viaje de los hogares y de la propiedad de vehículos en Alemania durante el periodo 1996 - 2006. Los resultados muestran que las características socioeconómicas de los hogares, la accesibilidad 
para el transporte público y la existencia cercana de centro comerciales o de esparcimiento y espacios de estacionamiento tienen efectos significativos en la posesión de vehículos. Específicamente hablando del ingreso, los autores reportan esta variable con periodicidad mensual y su impacto estimado alcanzó un valor de 0.103.

También se pueden encontrar estudios en los que se hallaron impactos negativos del ingreso en la propiedad de autos, Yagi y Managi (2016), reportan ocho regresiones para propiedad de vehículos en Japón entre 1980 y 2009, las regresiones están diferenciadas por tamaño del automóvil desde auto compacto hasta automóvil grande, los coeficientes estimados oscilan entre -0.102 para los carros más grandes hasta 0.122 para los más pequeños, todos los coeficientes son estadísticamente significativos. En este mismo sentido se encuentra el trabajo de Eakins (2013), que utiliza dos modelos, un logit multinomial y un logit ordenado, para estimar el tipo de relación que presentan el ingreso y la posesión de vehículos. Los hallazgos de este autor indican que el ingreso por adulto impacta de diferente manera a la tenencia de automóviles. Las estimaciones resultan negativas cuando los hogares tienen cero y un auto y positivas cuando los hogares tienen dos y tres autos o más.

Existen estudios en los que se utilizan modelos probabilísticos o de otro tipo para estimar la relación entre ingreso y tenencia vehicular. Estudios como el de Abu et al., (2016), cuantifica el impacto de diversas variables en la propiedad de vehículos en Holanda en el que se utilizó una regresión logística. El estudio reporta una gran cantidad de variables incluyendo la etnicidad de la población. Respecto del ingreso, los autores dividen la muestra en cuartiles. Los cuartiles de ingreso más bajo presentan un menor impacto en la propiedad de vehículos mientras que el cuartil de ingreso más alto reporta un impacto positivo y significativo.

Como se pudo observar, la mayoría de la literatura reporta que el ingreso y la posesión vehicular se relacionan positivamente, no obstante, no debe perderse de vista que no siempre resulta así. Los estudios que encontraron relaciones negativas tuvieron la característica contar con información desagregada sobre el número de automóviles por hogar.

Una variable que resulta común encontrar en los estudios con información agregada que buscan los determinantes de la propiedad de automóviles es el número de adultos por hogar, la justificación de la introducción de esta variable es que los adultos son lo que generan la movilidad pues demandan viajes para llevar a cabo su vida cotidiana. Los estudios que utilizan Mínimos Cuadrados Ordinarios (MCO) se mencionarán en primer lugar para después dar paso a los que utilizan otra herramienta.

Dargay y Giuliano (2006), obtienen rangos de adultos por hogar, el coeficiente asociado al menor rango es negativo y significativo (-I.2I) ésta estimación es la única 
de signo negativo encontrado en la literatura. Por su parte, el impacto del rango de mayor cantidad de adultos por hogar es positivo y significativo $(0.39)$. Otra estimación hecha por Dargay (200I), encontró que la elasticidad fue 0.243 cuyo valor fue significativo.

Dentro de los que no utilizan técnicas estándar está el estudio de Dargay (2002), quien halla que la cantidad de adultos impacta positivamente la propiedad de vehículos, el coeficiente reportado es de 0.6I. Por último, Abu et al., (2016), estima los impactos de la presencia de adultos en la propiedad con una regresión logística, sus diferentes modelos tienen coeficientes cuyo valor va de 0.77 hasta 1.55 , todos positivos y estadísticamente significativos. En algunos estudios como el de Hong y Eungcheol (2004) los adultos se pueden asociar al número de licencias de manejo expedidas bajo el entendido de que una persona adulta puede obtener una licencia de manejo, al tomarse como variable independiente. Las licencias mostraron un impacto positivo y significativo en la posesión de automóviles. Como pudo observarse, la mayoría de la literatura revisada reporta que una mayor cantidad de adultos se asocia positivamente con una mayor posesión de automóviles.

La tercera variable que se revisará y que ha sido utilizada por la literatura como determinante de la propiedad de vehículos es la existencia de vialidades y carreteras. La relación se da cuando la construcción de una carretera o vialidad genera una disminución en los costos de transportación de los agentes económicos incentivando la compra o adquisición de un vehículo automotor. Este fenómeno recibe el nombre de tráfico inducido. Algunas de las primeras mediciones fueron hechas por Goodwin (1996), quien indica que un aumento en los kilómetros de caminos induce un Io por ciento del volumen de tráfico en el corto plazo y un 20 por ciento en el largo plazo.

Para el caso de México, Galindo (2006:123), confirma la existencia de este fenómeno indicando que la elasticidad de las vialidades al consumo de gasolina, variable proxy del uso de vehículos automotor, es de 0.15. La estimación es estadísticamente significativa ${ }^{3}$. Huang (20II), encuentra una relación positiva entre dichas variables, el autor utiliza mínimos cuadrados ordinarios y cointegración para realizar sus estimaciones y halla significancia estadística. El coeficiente estimado tomó un valor de 0.278 que además resultó significativo. Por su parte, Sun et al., (2015), encuentra que la relación entre ambas variables es positiva, aunque su estimación no tiene

3 Se debe tener cuidado pues el volumen de tráfico se puede interpretar como uso de automóvil en lugar de posesión que no son lo mismo, pero sí son conceptos estrechamente relacionados. 
significancia estadística, el autor utilizó como variable kilómetros recorridos por persona dentro de cada territorio.

La cuarta variable por analizar es la densidad de población. Se debe tomar con cuidado puesto que es una variable en la que la literatura ha encontrado resultados no definitivos, de hecho, es la variable que arroja resultados más heterogéneos respecto de sus impactos estimados.

En el estudio de Sun et al., (2015), la densidad de población resulta ser la variable de mayor impacto en la propiedad, la estimación resultó negativa y significativa $(-0.422)$, en ningún otro documento se encontró que esta variable haya tenido el mayor impacto. Yagi y Managi (2016), usan regresiones por tamaño de automóvil y encuentran que para regresiones asociadas a automóviles más grandes los coeficientes toman valores desde -6.76I y de 5.546 para los automóviles compactos. Ambas estimaciones tienen significancia estadística.

Dargay y Giuliano (2006), dividen en tres rangos la densidad de población en los cuales el menor impacta positivamente a la propiedad de automóvil, el coeficiente estimado toma un valor de 0.17 y es estadísticamente significativo. No obstante, los dos rangos superiores tienen impactos negativos y significativos con valores de -0.09 y -I.18 respectivamente. Abu et al., (2016), divide la densidad en 4 rangos, el impacto en la propiedad de vehículos oscila entre 2.02 para la densidad más baja y 0.94 para la más alta siendo ambas estimaciones positivas y significativas. Para el caso de Osaka, Japón y Kuala Lumpur, Malasia, el estudio de Yamamoto (2009), reporta que la densidad de población mostró impactos negativos y significativos para ambas ciudades, aunque el efecto fue más grande para Osaka.

La quinta variable es la presencia de transporte público. Como se mencionó en párrafos anteriores, la existencia de este tipo de transporte puede ser un sustituto de la posesión de automóviles. No obstante, su impacto ha mostrado ser ambiguo en la tenencia de carros. Sun et al., (2015), encuentra que el impacto del transporte público es positivo (0.0433) pero no presenta significancia. Eakins (2013), mide la variable transporte público como el gasto que realizan los ciudadanos en este tipo de modalidad reportando que cuando un hogar no tiene automóviles o cuando tiene uno solo, el aumento en el gasto que realizan los usuarios en transporte público, por ejemplo por una subida de la tarifa, tiene como resultado un incremento en la propiedad de vehículos, pero a medida que aumenta el número de automóviles en los hogares el coeficiente se vuelve negativo, todas sus estimaciones resultan estadísticamente significativas.

Desde el estudio de Kitamura (1989), se mostró que aumentos en la posesión y uso de autos pueden no ser sustituido con mejoras en el transporte público. Culli- 
nane (2002), encontró que las políticas públicas que han tratado de hacer atractivo el uso de transporte público para desalentar la posesión de vehículos han sido muy poco efectivas, pero en ciudades como Hong Kong donde el transporte público es abundante y barato, la posesión y uso del automóvil es muy baja implicando que si el transporte público no tiene calidad se incentiva la posesión y el uso pues el usuario lo preferirá para realizar sus traslados. Eso significa que un signo positivo en la relación transporte público posesión de vehículos indicaría una mala calidad en el transporte público y uno negativo, buena calidad.

Woldeamanuel et al., (2009), halla que el tipo de servicio que ofrece el transporte público impacta negativamente a la posesión, además se encontró que el coeficiente fue significativo (-0.14I). Por su parte, el estudio Yagi y Managi (2016), encuentran que el tamaño del automóvil se ve impactado de manera diferente por el transporte público. Cuando se toman en cuenta autos pequeños el transporte público impacta negativamente su propiedad con coeficientes que toman valores de -0.059 y - -0.030 y significativos, pero cuando se toman autos de mayor tamaño se vuelve positivo (o.098) y significativo.

Para Woldeamanuel et al., (2009), la variable asociada al uso de transporte público tuvo un coeficiente negativo de -0.I4I. Otro hallazgo de interés en Woldeamanuel apunta a que las políticas que se implementaron en Alemania para mejorar la infraestructura peatonal y de bicicletas pudieron haber contribuido a los cambios de los comportamientos de viajes de los hogares. Cabe resaltar que la variable de mayor impacto en este estudio resultó ser el tamaño del hogar.

\section{Algunos hechos estilizados}

En esta sección se analizan algunos de los datos existentes para tener un panorama del comportamiento de la magnitud de la posesión de vehículos en los municipios de México.

Durante el año 2010 se registraron 20, 472,889. Las tres zonas metropolitanas con mayor número de vehículos son la Zona Metropolitana del Valle de México con 5,931,832, le sigue la Zona Metropolitana de Guadalajara con I,799,356 y la Zona Metropolitana de Monterrey con I,780,380. No se debe perder de vista que la agregación de la tasa de automóviles a nivel nacional puede no reflejar puntualmente lo que sucede en las grandes urbes o zonas poco pobladas.

$\mathrm{El}$ análisis de los datos revela algunos aspectos importantes que no deben dejarse de lado. Por ejemplo, la tasa de automóviles por cada ıoo,00o mil habitantes ha ido 
incrementando a nivel nacional hasta casi cuadruplicarse en el periodo que va desde I980 hasta 2014 como puede apreciarse en la gráfica I. La tasa media de crecimiento anual para los datos graficados es de $2.71 \%$. En este punto cabe detenerse a hablar del nivel de saturación vehicular que de acuerdo con Dargay et al., (2007), difiere en cada país dependiendo de su ingreso, la densidad de población, de la edad de la población y del porcentaje de población urbana. El autor estima un punto de saturación para países de la OCDE, el punto más alto lo obtuvo Estados Unidos que podría alcanzar dicho nivel al llegar a 852 autos por cada Iooo habitantes mientras que para México es de 840 .

\section{Gráfica I.}

Evolución de la tasa de automóviles a nivel nacional 1990-2014

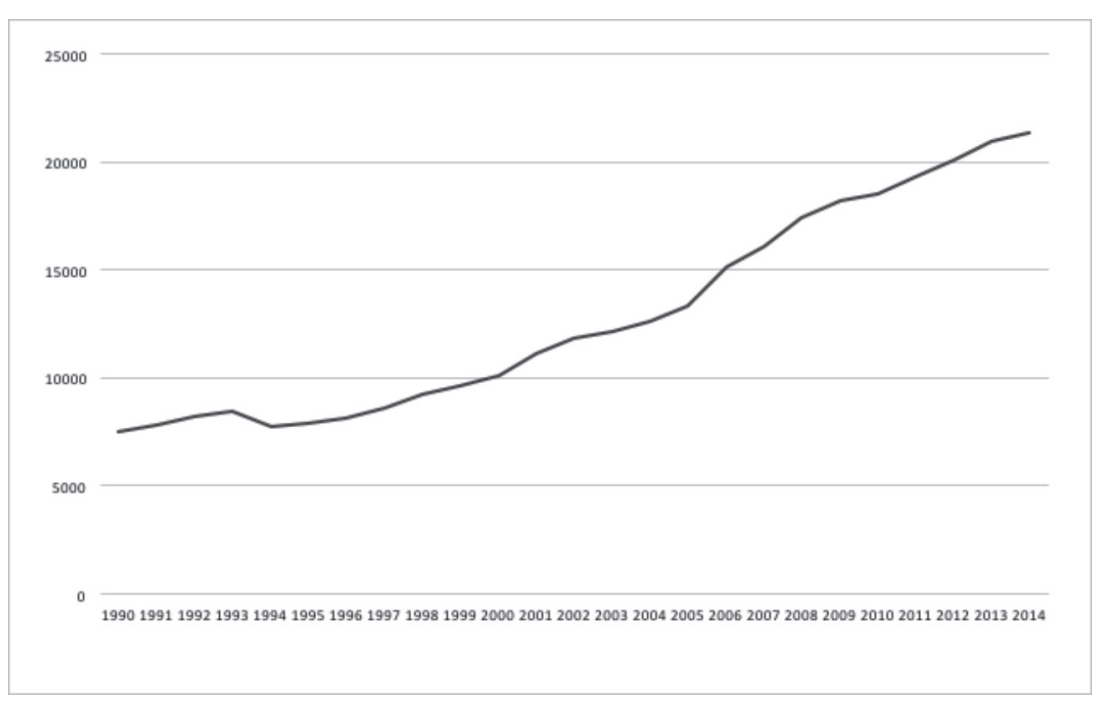

Fuente: elaboración propia con datos de INEGI. Censos económicos 2010

Para facilitar la interpretación visual se incluye el mapa i en el que se puede apreciar la distribución municipal de la posesión de automóviles sobre el territorio nacional dividido en quintiles. Los colores oscuros representan los territorios en los que la tasa de automóviles por cada roo mil habitantes es mayor y los colores claros en los que es menor.

Se puede apreciar que las zonas en las que se concentra la mayor tasa de automóviles se encuentra en municipios urbanos como puede ser la Ciudad de México, Guadalajara, Monterrey y Cancún. Llama la atención las aglomeraciones existentes 
de municipios con altas tasa de posesión de vehículos en toda la península de Baja California, así como las zonas fronterizas de Sonora, Tamaulipas, Chihuahua, la zona costera de Sinaloa, Norte de Nuevo León y el oeste de Sonora. De la misma forma, hay aglomeraciones de municipios con tasas bajas de posesión que se localizan mayoritariamente en los estados de Chiapas, Oaxaca, Yucatán, Campeche y focalizada en el sureste de Chihuahua y municipios fronterizos de Nayarit y Durango, así como el occidente de Coahuila que resultan ser lugares que no se encuentran cerca de aglomeraciones urbanas. En los estados de Chiapas, Oaxaca y Yucatán resalta que sus capitales presentan altas tasas de posesión aislándose del resto de los municipios que presentan bajas tasas de posesión vehicular.

Mapa I.

Distribución municipal de la tasa de posesión de automóviles 2010

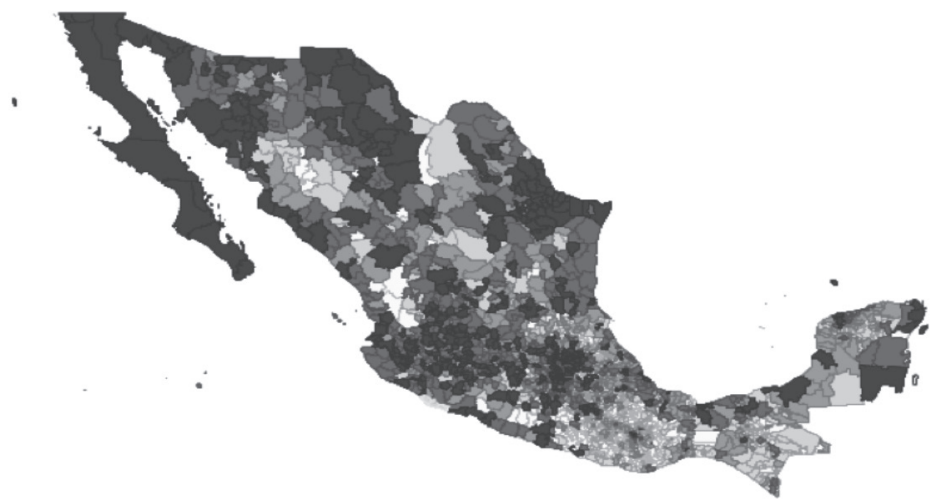

Fuente: elaboración propia con datos de INEGI. Censo económico 2010

En este punto cabe detenerse a reflexionar sobre el problema de las unidades de área modificable (MAUP) ${ }^{4}$ que es una situación que se presenta cuando se trabajan datos agregados referenciados a una unidad administrativa. El problema de las unidades de área modificable se relaciona con el hecho de que las medidas para datos transversales son sensibles a la agregación y también a las combinaciones de unidades adyacentes. Derivado de ello, este problema tiene dos divisiones: el efecto escala y la división de zonas. El efecto a escala indica que los resultados de algún análisis pueden cambiar si se tiene la misma variable a diferente escala de medición

4 Por sus siglas en inglés Modifable Areal Unit Problem 
mientras que la división de zonas puede provocar que la reagrupación de los datos en diferentes zonas se obtengan resultados distintos. El problema de fondo es que se puede concluir que el comportamiento agregado es el mismo que el individual. En pocas palabras, se debe tener cuidado con los resultados pues, de contar con otro tipo de agregación, los resultados podrían variar.

\section{Análisis econométrico}

La revisión de la literatura arrojó que son diversas las variables que inciden en el comportamiento de la posesión de automóviles. En esta sección se estimarán los impactos estudiados para México a nivel municipal con datos del censo económico 2010 en cuya estructura se reportan los vehículos automotores registrados que, a su vez, se desagregan en automóviles, motocicletas, camiones para pasajeros y camiones para carga. Se toma como posesión de vehículos al hecho de que los automóviles reportados pertenecen a algún agente económico ya sean públicos o privados y, en ese sentido, son posesión de una persona o familia o empresa y en el caso de ser públicos, son propiedad de alguna dependencia de gobierno.

Se presentarán tres modelos que tienen la característica de estar divididos en dos diferentes ambientes que son el urbano y el rural ${ }^{5}$, además de presentar el modelo que engloba a todas las observaciones. La literatura mostró que la división entre territorios urbanos y rurales puede arrojar resultados más confiables.

La variable dependiente reportada en los primeros dos modelos -total de municipios y municipios urbanos- es la tasa de automóviles por cada Ioo mil habitantes obtenida del censo de población y vivienda $2010^{6}$. Cabe aclarar que, con el objetivo de obtener el mejor ajuste, a los tres modelos se les aplicó logaritmo tanto a la variable dependiente como a las independientes. La estimación se llevó a cabo usando mínimos cuadrados ordinarios (MCO) que es una técnica que busca obtener estimadores que minimice la suma del cuadrado de los residuos. Los resultados de esta técnica tienen la particularidad de que los coeficientes hallados miden el

5 Para distinguir lo urbanos de lo rurales se utilizó la clasificación que ofrece el INEGI en la que se tipifica que lo urbano obedece a municipios en los que la población superior a 2,500 habitantes y en lo rural la población es menor a dicha cantidad.

6 Es necesario apuntar las limitaciones de tener información en solo año pues no se podrán capturar efectos intertemporales y efectos de experiencia como los que se pueden estudiar cuando existen datos en forma de panel. Una profundización al respecto puede verse en Hanly y Dargay (2000). 
efecto que tienen las variables dependientes sobre la variable control, en este caso, la posesión de automóviles. Además, la linealidad asociada al modelo implica que, sin importar el valor de las variables independientes, una modificación unitaria en éstas variable siempre tendrá el mismo efecto en la variable de control. El uso de MCO está recomendado para modelos de una sola ecuación (Morales, 200I:295) por lo que se consideró que esta técnica era adecuada.

Por el lado de las variables independientes se debe mencionar que la densidad de población se midió de la manera usual, es decir, el número de habitantes sobre la extensión del territorio del municipio. El ingreso es aproximado por la escolaridad promedio de los habitantes de cada municipio. Las vialidades son representadas por la longitud en kilómetros que presentó el municipio en el año 20Io, esta variable se tomó de la publicación de INEGi llamada Infraestructura y Características del Entorno Urbano 20ı. El transporte público está representado por el número de camiones de transporte público de pasajeros que operan en el municipio en el que está registrado. Por último, la población adulta se representa con el total de adultos por municipio.

TABLA I.

Resultados de los modelos estimados

\begin{tabular}{lccc}
\hline & $\begin{array}{c}\text { Total } \\
\text { Municipios } \\
(\mathrm{I})\end{array}$ & $\begin{array}{c}\text { Municipios } \\
\text { Urbanos } \\
(2)\end{array}$ & $\begin{array}{c}\text { Municipios } \\
\text { Rurales } \\
(3)\end{array}$ \\
\hline Constante & $\begin{array}{c}-9.922^{*} \\
(\mathrm{I} .0646)\end{array}$ & $\begin{array}{c}-8.852^{*} \\
(0.9357)\end{array}$ & $\begin{array}{c}-3.2502^{*} \\
(0.489)\end{array}$ \\
\hline Ingreso & $2.7059^{*}$ & $2.5085^{*}$ & $1.009^{*}$ \\
\hline Densidad Poblacional & $(0.199 \mathrm{I})$ & $(0.1866)$ & $(0.0599)$ \\
\hline Transporte Público & $0.5527^{*}$ & $0.3011^{*}$ & $0.1107^{*}$ \\
& $(0.048 \mathrm{I})$ & $(0.0423)$ & $(0.024)$ \\
\hline Población Adulta & $0.0818 \mathrm{I}^{*}$ & $0.0824^{*}$ & 0.2009 \\
& $(0.0197)$ & $(0.0177)$ & $(0.6502)$ \\
\hline Vialidades & 0.3705 & $1.063^{*}$ & $0.021^{*}$ \\
& $(0.3167)$ & $(0.2865)$ & $(0.0036)$ \\
\hline
\end{tabular}




\begin{tabular}{lccc}
\hline $\begin{array}{l}\text { Heteroscedasticidad } \\
\text { Breusch-Pagan test }\end{array}$ & $0.078^{*}$ & $0.0928^{*}$ & 0.0000 \\
\hline Normalidad Swilk test & $0.1866^{*}$ & $0.7162^{*}$ & 0.0006 \\
\hline Ramsey test & $0.4059^{*}$ & $0.5543^{*}$ & $0.2501^{*}$ \\
\hline $\mathbf{R}^{2}$ & 0.9147 & 0.8702 & 0.3988 \\
\hline
\end{tabular}

Error estándar entre paréntesis * Estadísticamente significativo al $5 \%$

En la tabla i se presentan los resultados de las estimaciones. En los modelos referentes a los municipios urbanos y todos los municipios se observa que las estimaciones son positivas y estadísticamente significativas además de pasar satisfactoriamente las pruebas de diagnóstico ${ }^{7}$. El coeficiente de determinación resulta alto para los primeros dos modelos implicando que la proporción de variación de la variable dependiente es satisfactoria.

La variable de mayor impacto para modelos uno y dos es el ingreso. Para el caso del modelo que toma los municipios urbanos, la elasticidad hallada es positiva $y$ toma un valor muy alto (2.508) indicando una gran sensibilidad de la posesión de automóviles al ingreso. El signo hallado coincide con Sun et al., (2015) e Ingram y Liu (1999). Este resultado podría ser parte de la explicación del por qué algunas de las ciudades del país como la Ciudad de México y Guadalajara aparecen en los primeros lugares en los índices internacionales que miden el volumen de tráfico en grandes urbes ${ }^{8}$. De la misma forma, la elasticidad correspondiente al modelo que engloba todos los municipios alcanzó un valor de 2.7059 que es la elasticidad más grande encontrada en la literatura revisada.

Otra variable que mostró tener un fuerte impacto en la posesión vehicular fue la población adulta cuyo coeficiente superó la unidad ( $\mathrm{I} .063)$ indicando sensibilidad de la posesión a este tipo de población lo que está asociado directamente con la estruc-

7 No se presentaron correlaciones que indiquen existencia de multicolinealidad entre las variables explicativas.

8 Existen varios índices que miden el tráfico vehicular en ciudades alrededor del mundo, en particular el Magnatec índex publicado en 20I4, mediante su metodología stopstart, encontró que la Ciudad de México se posicionó en el tercer lugar de las ciudades con mayor tráfico del mundo mientras que Guadalajara se fue el noveno. Otros índices como el Commuter Pain Índex publicado en 2ori encontró que la Ciudad de México fue la que generó la mayor congestión vehicular en el mundo, en dicho estudio la ciudad de Guadalajara no obtuvo un lugar en las primeras 20 posiciones. Otro índice conocido como Tom Tom Traffic Index publicado en 2016, coincidió en posicionar a la Ciudad de México como el primer lugar en congestión vehicular en el mundo. 
tura de edades de la población de los municipios urbanos que se puede prever que siga creciendo. Los resultados son consistentes con Dargay (200I), Dargay (2002) y Dargay y Guliano (2006). Este resultado confirma que las necesidades de movimiento al interior de una ciudad son llevadas a cabo en buena medida por adultos. En el modelo que engloba todos los municipios esta variable no tuvo significancia estadística. En este punto cabe detenerse a reflexionar sobre la estadística disponible respecto de esta variable. La encuesta nacional de la juventud 20 Io reveló que $67 \%$ de las personas entre 20 y 29 años vivían con sus padres, esto significa que los adultos de esas edades aparte de los padres viven en una misma casa implicando que la cantidad de adultos por hogar ha incrementado y dadas las razones por las cuales los adultos no viven solos se puede esperar que esta variable siga incrementando generando el impacto encontrado en la posesión de vehículos.

El signo encontrado en la estimación correspondiente a transporte público fue positivo indicando que un aumento en la cantidad de vehículos de transporte público incentiva la posesión de automóviles privados. Este resultado coincidió con Sun et al., (2015), los menores niveles de ingreso en Eakins (2013) y la existencia de automóviles de mayor tamaño en Yagi y Managi (2016). Como se apuntó en el primer apartado esta situación es evidencia de mala calidad en el transporte público urbano. Esto significa que los habitantes no consideran como un sustituto al transporte público y prefieren el automóvil ${ }^{9}$.

La extensión de las vialidades impactó positivamente a la posesión coincidiendo con Huang (20II) y Sun et al., (2015). Esta relación apoya la evidencia de tráfico inducido encontrada para México por Galindo (2006). Para ambos modelos -total de municipios y municipios urbanos- el aumento es menos que proporcional.

En el modelo correspondiente al ámbito rural se puede observar que se registraron coeficientes estadísticamente significativos y positivos. No obstante, no pasó las pruebas de heterocedasticidad ni normalidad. Se probaron modelos logarítmico-lineales para buscar un mejor ajuste; también se modificó la manera de medir la variable dependiente utilizando el número agregado de autos y motocicletas sobre el total de kilómetros cuadrados del municipio tanto en logaritmos como en niveles, pero no mejoraron los resultados. Alternativamente se usaron variables binarias para controlar los municipios muy poco poblados, pero tampoco se tuvo éxito por lo que se decidió dejar su modelación para una investigación posterior y más profunda.

La mala calidad del transporte público en México está reportada en diversa bibliografía a lo largo del tiempo. Al respecto puede verse Boltnivik (1984:35) y más recientemente Sánchez y Romero (2010: 62). 


\section{Conclusiones}

El objetivo de este artículo fue cuantificar los determinantes de la posesión de vehículos en los municipios de México en el año 2010 que se compararon con los que ofrece la evidencia internacional. La revisión de la literatura que versa sobre el tema arrojó que han sido utilizadas diversas variables que impactan la posesión de automóviles, además de que hay variaciones en la magnitud de los impactos, los signos encontrados y la significancia estadística de los mismos.

La información permitió separar municipios urbanos y rurales que se justifica por la diferencia de población y, por tanto, de movilidad y estimar los determinantes separadamente. Además, se estimó un modelo con todas las observaciones que permitió observar su comportamiento general.

Los resultados de los modelos para municipios urbanos y para la totalidad de la muestra son similares mientras que el modelo correspondiente a municipios rurales presentó problemas con las pruebas de especificación. Las variables incluidas como independientes fueron ingreso, densidad de población, vialidades, población adulta y transporte público. Para el modelo que tomó los municipios urbanos las estimaciones arrojaron que la variable de mayor impacto fue el ingreso cuya elasticidad fue positiva y significativa. La magnitud del resultado supera a todas las encontradas en la literatura indicando que la propiedad de vehículos es altamente sensible a cambios en el ingreso. Otro resultado de importancia es la evidencia que sugiere la existencia de tráfico inducido pues la reacción de la propiedad de automóviles a las vialidades fue positiva y significativa mientras que el transporte público mostró un impacto positivo en la propiedad lo que, de acuerdo a la evidencia internacional, se interpretó como una mala calidad en el transporte público. Por su parte, la población adulta impacta positivamente a la propiedad de vehículos por lo que, dada la situación de la pirámide poblacional, se puede esperar que la posesión vehicular siga incrementando.

La discusión respecto de la recomendación para desarrollar políticas públicas que se pueden desprender de este estudio se refiere a las variables provisión de vialidades y transporte público pues en ellas el gobierno, mediante políticas públicas, puede modificar su comportamiento. Dado que en las zonas urbanas las externalidades derivadas de la presencia de automóviles se presentan con mayor intensidad, se hace necesaria la utilización de políticas.

Las estimaciones revelan que la provisión de vialidades afecta positivamente a la posesión de automóviles. Si la posesión se toma como proxy del uso de automóvil los resultados del modelo se pueden interpretar como evidencia de tráfico 
inducido. Como lo afirma Galindo (2006: 13I), el tráfico inducido implica que la construcción o mejoramiento de infraestructura vial genera una demanda creciente de viajes que eleva el tráfico. De manera que si el gobierno no quiere incentivar un mayor número de viajes y congestionamiento debe entender que construir vialidades generará más tráfico surgiendo como alternativa la generación de políticas de transporte no motorizado como el uso de la bicicleta que tiene la ventaja de no generar emisiones de gases contaminantes y, al mismo tiempo, puede servir como política para combatir la obesidad.

La segunda variable sobre la que se puede llevar a cabo algún tipo de política es la provisión de transporte público, en particular sobre su calidad. Las políticas se deben encaminar a mejorar sustancialmente diversos aspectos del transporte público como la eficiencia, tarifa, seguridad y comodidad de manera que desincentive la posesión y / o uso de automóviles. De acuerdo con Caudillo, et al (2016), existe evidencia empírica sobre la relación entre entorno construido y comportamiento de viajes en la Zona Metropolitana del Valle de México. A través de diferentes modelos de regresión se encuentra que hay una oportunidad marginal para influir en el comportamiento de viajes no laborales motorizados. Es decir, existe una oportunidad para influir sobre algún tipo de viajes.

\section{Bibliografía}

Abu, Toasin Md Oakil,, Akil, Dorien. y Nijland, Hans (2016),"Determinants of car ownership among young households in the Netherland: The role of Urbanization and Demographic and economic characteristics", Journal of Transport Geography, 51, pp. 229-235.

Bagley, Michael y Mokhtarian, Patricia (2002),"The impact of residential neighborhood type in travel behavior: a structural equation modeling approach", Annals of Regional Science, vol. 36, núm. 2. pp. 279-297.

Boltnivik, Julio (1984), "Satisfacción desigual de las necesidades esenciales en México", en: Cordera, Rolando y Tello, Carlos. La desigualdad en México, siglo xxi editores, México

Cao, Xinyu, Handy, Susan y Mokhtarian, Patricia (2006), "The influences of the built enviroment and residential self-selection on pedestrian behaviour: Evidence from Austin", tx, Transportation 33, pp. I-20. 
Caudillo, Cos., Camilo, Montejano y Jorge, Schmidt y Mura, Suyay (2016), La movilidad en la sociedad contemporánea, Conference Paper, Centro de estudios demográficos, urbanos y ambientales. El Colegio de México.

Clark, Stephen (2009), "Characterising and predicting car ownership using rough sets", Transportation Research Part C, Volumen I7, pp. 38I-393.

Chingcuanco, Franco y Miller, Eric (2014),"A meta-model of vehicle ownership choice parameters", Transportation, Volumen 4I, pp. 923-945.

Cullinane, Sharon (2002), "The relationship between car owenership and public transport provision: a case study of Hong Kong", Transport Policy, Vol. 9, núm. I, pp. 29-39

Dargay, Joyce y Gately, Dermot (1999)."Incomés effect on car and vehicle ownership, world wide: 1960-2015", Transportation Research Part A.

Dargay, Joyce (200I),"The effect of income on car ownership: evidence of asymmetry", Transportation Research Part A: Policy and Practice, vol. 35 issue 9, pp. 807-82I.

Dargay, Joyce (2002), "Determinants of car ownership in rural and urban areas: a pseudo-panl analysis", Transportation Research Part E 38, pp. 351-366

Dargay, Joyce y Guliano, Genevieve (2006), “Car ownership, travel and land use: a comparision of the us and Great Britain", Transportation Research Part A: Policy and Practice, Vol. 40, núm. 2, pp. I06-I24.

Dargay, Joyce, Dermot, Gately y Sommer, Martin(2006),"Vehicle Ownership and Income Growth, Worldwide: 1960-2030", Energy Journal, vol. 28, núm. 4, pp. I-32.

Eakins, John (2013), The determinants of Household car ownership: empirical evidence from the Irish household budget survey. SEEDS I44, University of Surrey.

Galindo, Luis, Heres, David, y Sánchez, Luis (2006), “Tráfico inducido en México: contribuciones al debate e implicaciones de política pública", Estudios demográficos y urbanos, vol. 2I, núm, pp. 123-157.

González-Arellano, Salomón (2013), "El sistema automóvil: propuesta conceptual a partir de la noción de operador espacial", Quivera, Vol. 15, núm. 2013-2, julio-diciembre, pp. 23-42.

Goodwin, Phil (1996), "Empirical evidence on induced traffic: A review and synthesis", Transportation, vol. 23, núm 23.pp. 35-54.

Guerra, Erick (2015), "The geography of car ownership in Mexico City: a joint model of housholds residential location and car ownership decicsions", Journal of Transport Geography, vol. 43, pp. 17I-I80. 
Handy, Susan, Cao, Xinyu y Mokhtarian Patricia (2005), "Correlation or causality between the buitl enviroment and travel behavior? Evidence from Nothern California", Transportation Research Part D, Io, pp. 427-444. Hanly, Mark y Dargay, Joyce (2000), "Car ownership in Great Bretain, a panel data analysis", Transportation Research Board, Vol. 1718, núm, I. pp.83-89. Hong, Sok Kim y Eungcheol, Kim (2004), "Effects of public transit on automobile ownership and use in households of the USA", Review of urban and regional development studies, vol. I6, núm. 3, pp. 245-262.

Hsu, Tien, Chia-Chia, Tsai y Lin, Yu (2007),"Comparative analysis of household car and motorcycle ownership characteristics", Journal of Eastern Asia society for Transport studies, vol. 7, pp. 105-II5.

Huang, Xiayi (20II), Car Ownwership Modeling and forecasts for China. Mimeo, Tesis de Maestría. Michigan Technological University.

Ingram, Gregory y Liu, Zhi (1999), "Determinants of motorization and Road provision”, Policy Research Working Paper No. 2042, The World Bank. Instituto Nacional de Estadística, Geografía e Informática (2010), Encuesta Nacional de la Juventud.

Instituto Nacional de Estadística, Geografía e Informática (2015), Encuesta Nacional sobre Confianza del Consumidor. Documento metodológico. Johnstone, Nick, Serret, Ysé y Bureau, Clotilde (2009), The determinants of car ownership and use. OECD conference. Paris.

Kitamura, Ryuichi (1989), "A causal analysis of car ownership and transit use", Transportation, vol. 16, núm. 2, pp. 155-173.

Macfarland, Gregory, Garroe, Laurie y Mokhtarian, Patricia (2015), "The Influences of past and present residential locations on vehicle ownership decisions", Transport Research Part A, Vol. 74, pp. 186-200.

Matas, Ana y Raymond, Josep (2008). Changes in the structure of car ownership in Spain, Transportation Research A, vol. 42, núm. I, pp. 607-617.

Morales, Efraín (200I), Introducción a la econometría. Editorial ABYA-YALA, Quito, Ecuador.

Nobile, Agostino; Chandra, Bath, y Pas, Eric (1995), "A Random effects multinomial probit model of car ownership choice. National Institute of Statisitical Science", Technical Report Number 4I.

Potoglou, Dimitris y Kanaroglou, Pavlos (2008), "Modelling car ownership in urban areas: a case study of Hamilton, Canada", Journal of Transport Geography, Vol. I6, núm. I, pp. 42-54. 
Rooda, Matthew (1998), Toronto area car ownership study: A longitudinal survey and preliminary analysis of results. Masters degree Thesis. Mimeo Root, Amanda y Schintler, Laurie (1999),"Women, motorization and the environment", Transportation Research Part D, 4, pp. 353-355.

Ryan, James y Han, Gregory (1999), "Vehicle ownership model using family structure and accesibility: application to Honolulu Hawaii", Transportation Research Record: Journal of the Transportation Research Board, vol. 1676, pp. I-IO.

Sánchez-Flores, Oscar y Romero-Torres, Javier (2010), "Factores de Calidad del servicio en el transporte público de pasajeros: estudio de caso de la ciudad de Toluca, México", Economía, Sociedad y Territorio, vol. X, núm. 32, pp. 49-80.

Schwanen, Tim y Mokhtarian, Patricia. (2005), "What affects commute mode choice. Neighborhood physical structure or preference toward neighborhood", Journal of Transport Geography, vol. 13, pp. 83-99.

Sun, Bindog, Zhang, Tinglin, He, Zhou y Wang, Rui (2015), "Urban spatial structure and motorization in China", Journal of Regional Science. DOI:IO.IIII/jors.I2237

$\mathrm{Xu}$, Mingtao, Ye, Zhirui y Shan, Xiaofeng (2015), "Modelling, analysis and simulation of the codevelopment of road networks and vehicle ownership", Physica A, pp. I-12.

Yagi, Michiyuki y Managi, Shunsuke (2016),"Demographic determinants of car ownership in Japan", MPRA Paper No. 71614.

Yamamoto, Toshiyuki (2009),"Comparative Analysis of household car, motorcycle and bicycle ownership between Osaka metropolitan area, Japan and Kuala Lumpur, Malasya", Transportation, vol. 36, núm. 3, pp. 35I-366.

Woldeamanuel, Mintesnot, Cyganski, Rita, Schulz, Angelika y Justen, Andreas (2009),"Variation of households car ownership across time: application of a panel data model", Transportation, 36, pp. 371-387.

Resumen Curricular

Ignacio Javier Cruz Rodríguez es Doctor en economía por la Facultad de Estudios Superiores Acatlán, Universidad Nacional Autónoma de México. En docencia, ha impartido diversos cursos de licenciatura en FES Acatlán-UnAm y actualmente imparte el seminario en economía del transporte a nivel posgrado en el Centro de Investigaciones Socioeconómicas de la Universidad Autónoma de Coahuila. Es 
miembro del Sistema Nacional de investigadores. Publicaciones recientes: I. "Impactos Económicos de la salida del mercado de Mexicana de Aviación”(2017) UAdec, ISBN:9786075062884. "Un enfoque regional del autotransporte de carga en México: Teoría y aplicaciones. (2015), UAdeC. IsBN 9786075062440. 3. Aerolíneas de Bajo Costo en México: Competencia Modal, intermodal e intramodal. Contaduría y Administración (2012). Vól. 57, núm. 4. pp. 235-25I.

Citar como: Cruz Rodríguez, Ignacio Javier (2018), "Determinantes socioeconómicos de la posesión de automóviles en los municipios de México", Iztapalapa. Revista de Ciencias Sociales y Humanidades, núm. 84, año 39, enero-junio de 20I8, ISSN: 2007-9176; pp. I9I-2I2. Disponible en <http:// revistaiztapalapa.izt.uam.mx/index.php/izt/issue/archive >. 